\title{
Diverse Biological Behavior of Phyllodes Tumor of the Breast
}

\author{
Shekar Y Tati ${ }^{\mathrm{a}}$, Chidananda $\mathrm{KV}^{\mathrm{a}}$, Chaitanya Kumar Reddy $\mathrm{P}^{\mathrm{b}}$, Prashant $\mathrm{K}^{\mathrm{b}}$, \\ Harris Mohammed ${ }^{\mathrm{b}}$, Somdeep Ghosh ${ }^{\mathrm{b}}$, Paramesh $^{\mathrm{c}}$ \\ ${ }^{a}$.Professor, ${ }^{b}$. Post graduate, Department of General Surgery. \\ c, Professor, Department of Pathology, KVG Medical College \& Hospital, Sullia, DK, Karnataka, India.
}

\begin{abstract}
Phyllodes tumor is an uncommon fibro epithelial breast tumor with diverse biological behavior. The tumor closely resemble as fibroadenoma of the breast. On one end phyllodes is slow growing benign tumor, the other end tumor grows rapidly behave like sarcoma which metastasizes, and phyllodes can turn to frank sarcoma.

Tumor can be diagnosed on clinical basis i.e. large mass with lobulated (bosselated) surface; skin is thinned out due to stretching by rapidly enlarging tumor with engorged cutaneous veins. Lump usually present in upper and outer quadrant of the breast or occupies entire breast and freely mobile within the breast.

Final confirmation can be achieved by FNAC, USG and mammography and MRI. High recurrence rate is reported following limited resection where as wide excision has less recurrence. Simple mastectomy is indicated in case of large and recurrent tumor. Malignant tumor will not respond to chemo-radiotherapy.

We came across four cases of phyllodes tumors almost in a decade from 2004 to 2014. The age incidence was same as mentioned in the literature. Out of four cases two phyllodes turned to sarcoma. One was giant fibro adenoma in which a section of histological picture showed phyllodes element.

We are interested to publish this article because we observed the sequence of events in our cases as mentioned in the literature. Phyllodes tumor is a variant of fibroadenoma to benign phyllodes tumor, turning to sarcoma.
\end{abstract}

Keywords: Fine needle aspiration cytology (FNAC), Fibroadenoma, Phyllodes tumor, Sarcoma, Ultra sonogram (USG).

\section{Introduction}

Phyllodes, the word derived from Greek term phyllon, tumor looks as leaf like structure under microscope so the name phyllodes tumor was implied. The tumor was also called cystosarocoma phyllodes, cystosarcoma phylloides and serocystic disease of the Brodie (1). More than 60 terminologies were mentioned in the literature. WHO finalized and coined the term as phyllodes tumor to overcome the confusion of multiple terminologies (2).

Phyllodes tumor is an uncommon tumor accounts less than $0.5 \%$ of all benign breast diseases (1). The tumor is more common in elderly women between the age group of 35- 45 years but can occur in any age between $10-80$ years $(3,4)$.

Etiology of phyllodes tumor is unknown however giant fibro adenoma likely to turn phyllodes tumor. The tumor is typically large and fast growing, variable in consistency with lobulated surface and well defined borders. The skin over the breast is thin and shiny due to over stretching by rapidly enlarging tumor with engorged veins. The above description is a typical feature of phyllodes tumor not seen in any other breast conditions therefore phyllodes tumor can readily be diagnosed on clinical basis.

The tumor arises from peridutal stromal cells of the breast tissue. It is a fibro epithelial tumor, composed of epithelial and cellular stroma (1). On microscopic examination, papillary projections of tumor mass are seen inside the tumor seen as leaf like structure with occasional cystic spaces; therefore the name cystosarcoma phylloides was given.

Mammogram shows round opaque mass sometimes opaque lobules are seen within the large opaque mass $(4,5)$. Ultra sonogram will show multiple nodules within the hypo echoic mass lesion. There can be cystic spaces in the center of the nodule. Core biopsy is more useful than FNAC but most surgeons prefer excision biopsy since, clinical diagnosis is obvious.

Surgery is the treatment of the choice. Lumpectomy is indicated for small lesions. Moderate size tumor require wide excision (7) where as large benign or recurrent tumors require mastectomy which will clear the tumor with tumor free margin. Pre operative and post operative radiotherapy trials are going on as a part of breast conservative surgery (8). The results are awaited. 


\section{Material And Methods}

II.1. 45 year old female was presented with recurrent breast lump in her right breast with a past $\mathrm{h} / \mathrm{o}$ wide excision two years before for similar lump. On examination the recurrent lump was $15 \mathrm{X} 14 \mathrm{~cm}$ in size (fig.1). Surface was lobulated with well defined borders but irregular. The lump was having typical description of phyllodes tumor. Axillary lymph nodes were not palpable. Case was provisionally diagnosed as recurrent phyllodes and simple mastectomy was done. Histologically the tumor reported as phyllodes tumor with sarcomatous changes. After discharge patient was not available for follow up.

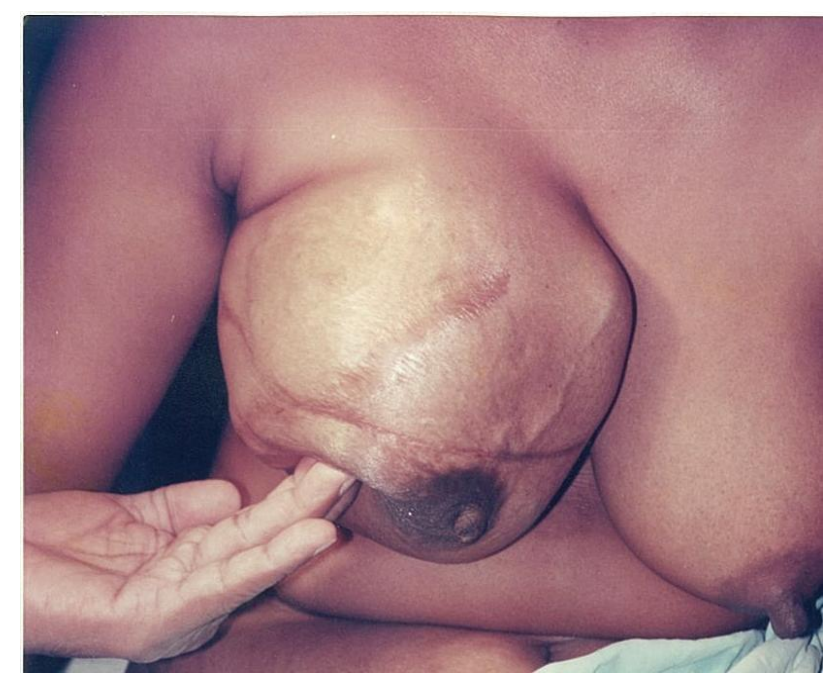

(Fig. 1) Right breast mass with previous scar with engorged veins

II.2. 40 year old female patient presented with lump in her right breast with a size of $10 \mathrm{X} 8 \mathrm{~cm}$ for one year duration with gradual progression without palpable lymph nodes in the axilla. The tumor was diagnosed as phyllodes tumor (fig. 2.a). Ultra sound was showing round heteroechoic masses with cystic spaces within the mass. Mammography was done, film was showing multiple hyper dense lobules were seen within the breast lump (fig. 2.b).Wide excision was done. After fascia dissected from the gross specimen the mass was having nodules on its anterior surface, posteriorly as single mass (fig.2c), Patient was followed for a year no recurrence was reported.

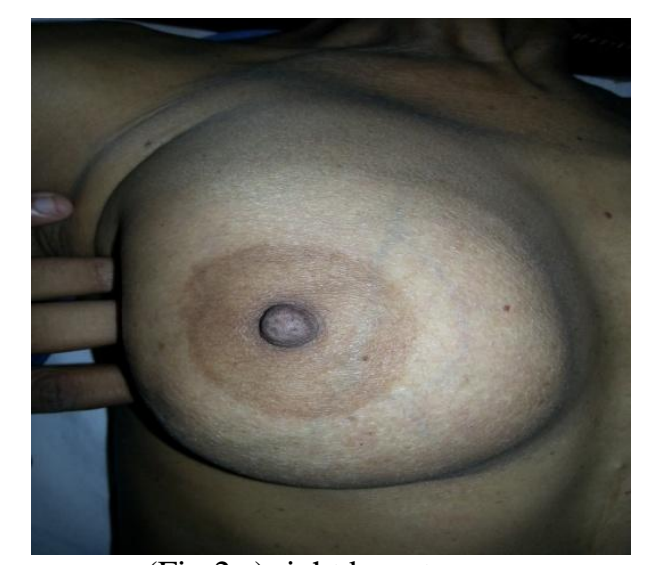

(Fig 2.a) right breast mass 


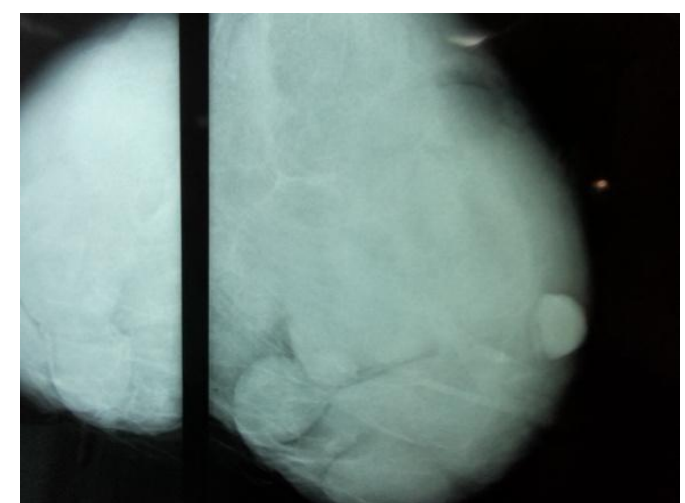

(Fig, 2.b) Mammogram showing multiple nodules within the mass lesion

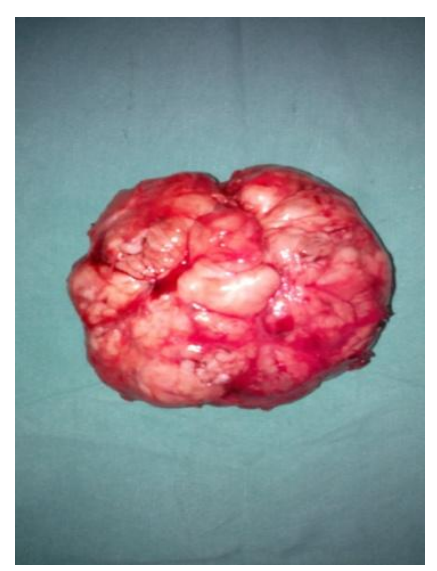

(Fig 2.c) Gross specimen showing anterior nodularity and posterior as single mass

II.3. 19 year old female presented with a lump in the left breast. The lump was $10 \mathrm{X} 8 \mathrm{~cm}$ in size with three lobules. The case was diagnosed as giant fibro adenoma with possibility of phyllodes tumor. Lumpectomy was done. Gross specimen was presented with three large lobules (Fig. 3) histologically it was reported as fibroadenoma but a part of the section was showing phyllodes element. Patient was followed for a period of six months, no recurrence was report.

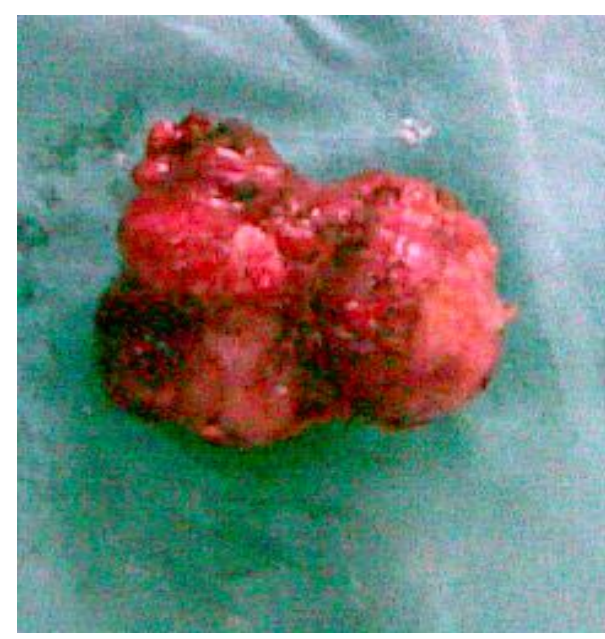

Fig, 3 showing three large lobules

II.4. 45 year old female presented with lump in her right breast for six months. The lump was rapidly increasing in size for three months with $\mathrm{h} / \mathrm{o}$ loss of appetite and loss of weight. She was known case of retroviral positive. The lump was $18 \times 15 \mathrm{~cm}$ in size, firm in consistency with bosselated surface. The skin over the breast was stretched with engorged veins. The nipple was pushed out and medially by tumor. Nipple and areola was excoriated with small multiple ulcers (fig.4a). it was diagnosed as pyllodes tumor and confirmed by FNAC. 
Simple mastectomy was done by taking universal precaution. Histological sections revealed phyllodes tumor with sarcomatous changes (fig. 4b). Patient advised for further treatment.

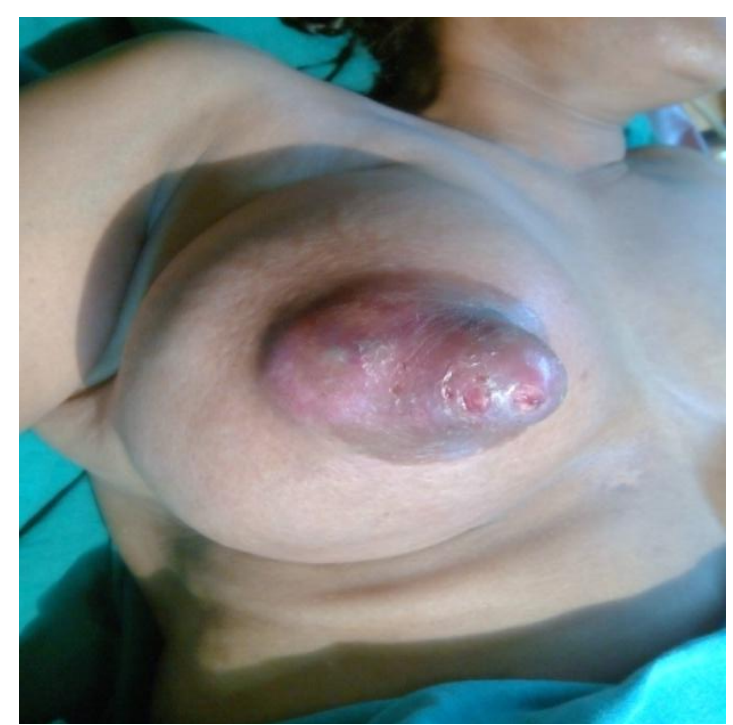

(Fig.4 a) showing large phyllodes tumor in the right breast

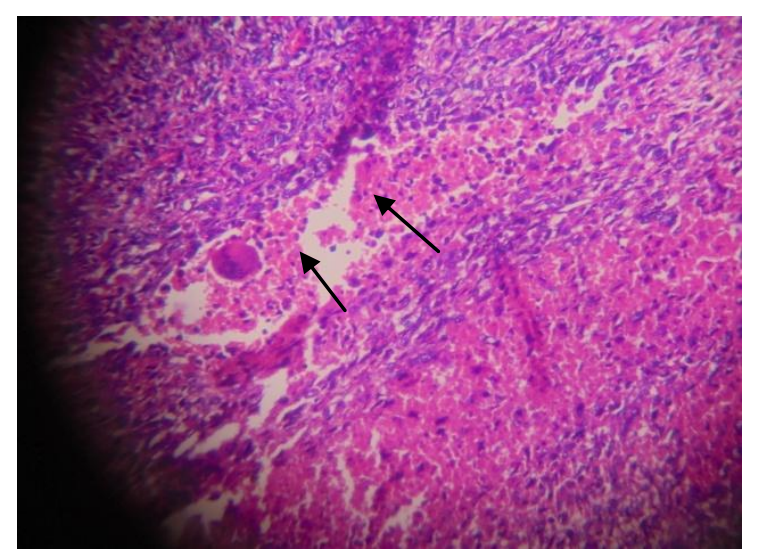

(Fig. 4b) showing Tumour cells arranged in fascicles with areas of necrosis and occasional tumour giant cells.

\section{Results}

Three of our four patient's age group was 40 and 45 years, the other one wass 19 years same as reported in the literature. Clinically thee elderly patients are presented with lump in the right breast. The lump was having classical feature of phyllodes i.e. large lobulated lump, freely moving within the breast with thinned out skin and engorged veins, the clinical entity of phyllodes tumors. The third one diagnosed as giant fibroadenoma but kept the possibility of phyllodes since lump was lobulated. It is unlikely that fibroadenoma is lobulated.

Histologically, two large tumors were reported as phyllodes tumor turning to sarcoma, one of two recurrent patients was retroviral positive patient. Tumor in younger patient was reported as fibroadenoma turning to phyllodes tumor. The above observation supports the view of diverse biological behavior of phyllodes tumor.

Therefore we conclude phyllodes tumor is more common in an elderly people, can occur in young individuals. As per our observation phyllodes tumor is more common in the right breast. It can easily be diagnosed clinically. Recurrent and large tumors will have high chance of turning to malignancy. Compromised immunity can also play a role in turning to sarcoma as one of our patients was retro viral positive. Mastectomy is the treatment of choice in such tumors. 


\section{Discussion}

Phyllodes term is derived from Greek term phyllon. It looks like a leaf under microscope, so the name phyllodes tumor was given. It was also called cystosaroma phyllodes the term described by Johannes Muller in 1838 (1) and cystosaroma phylloides and serocystic disease of Brodie. More than 60 termilogies are mentioned in the literature. World Health Organization (WHO) finalized the term as phyllodes tumor to overcome the confusion arising from multiple terminology $(1,2$, and 3$)$.

Phyllodes tumor is a spectrum of diseases consists of fibroadenoma, fibroadenoma variant, benign phyllodes, benign phyllodes with metastasis and frank sarcoma (1). In our cases we could observe this spectrum of sequence i.e. a phyllodes turning to sarcoma, a pure phyllodes and fibroadenoma turning to phyllodes tumor $(4,5)$. Phyllodes tumors are also classified as benign, border line and malignant phyllodes tumors.

Phyllodes tumor is a tumor of middle age group after forty years of age, usually before menopause. But tumor can present at any age ranging from 10 years to 80 years. (2). Phyllodes tumor can occur in a male who had previous gynecomastia (3). No known etiological or risk factors are identified for the development of phyllodes tumor.

\section{IV.1.Pathology}

Phyllodes tumor is large mass with multiple nodules on its anterior surface while posteriorly single mass (fig.2.c). The tumor is grayish white or reddish gray in color. Tumor arises from periductal stromal cells of the breast tissue

Histologically it is fibro epithelial tumor composed of an epithelial and cellular stroma. Papillary projections in the tumor mass are seen as leaf like structure with occasional cystic spaces, so the name cystosarcoma phyllode was given. Malignant potentiality is assessed on histological features of stromal cellularity, infiltration in to tumor margin and mitotic activity (4). The tumor may turn into pure sarcoma in $90 \%$ of the cases but in $10 \%$ cases biphasic presentation can be seen i.e. both sarcomatous and carcinomatous lesions $(6,7)$.

\section{IV.2. Clinical features,}

Phyllodes tumor basically is a tumor of elderly women, very few cases are reported in teen age groups. Our patients age group was 40 and 45 and 19 years old which is same as mentioned in the literature. Patient presents with firm painless lobulated lump mostly in upper and outer quadrant of the breast, with well defined borders. The lump is large in size and freely mobile within the breast without nipple retraction. When it is large the skin over the breast is thinned out with engorged veins and it is a diagnostic feature of phyllodes tumor. In case of large lump nipple is likely to be pushed out and deviated and there can be pressure necrosis resulting in ulceration as we could appreciate in our fourth case.

\section{IV.3. Investigations}

On mammogram film phyllodes tumor appears as a large round or oval mass with well-defined edge with rounded lobes inside the tumor $(5,6)$. We observed this finding in one case. Ultra sonogram will show multiple hypoechoic nodules within the cystic spaces in the center of the nodule. Core biopsy is more useful than FNAC but most of the surgeons diagnose the case on clinical ground go for excision biopsy.

\section{Treatment}

Surgery is main stay of the treatment. Wide local excision is the treatment of the choice in small benign tumors but wide local excision associate with high incidence of local recurrence $(8,9)$. Mastectomy is indicated in case of large and recurrent tumors and mastectomy will clear the tumor along with tumor margin.

Some reports are available in the literature for breast conservative surgery. Trials are going on where patient is given preoperative radiation, followed by surgical excision in the form of lumpectomy and again post operative radiotherapy for which the results are awaited $(5,8)$. Over all mastectomy is superior to beast conservative surgery since, it clears the entire tumor with tumor margin. Malignancy arising from phyllodes will not respond to radiotherapy or chemotherapy $(8,9)$.

\section{Conclusion}

Phyllodes tumor is more common in elderly patient but can occur from adolescent to old age. Right breast is more commonly involves. Clinical diagnosis is certain because of its classical. USG and mammography and core cut biopsy or FNAC will clinch the diagnosis for confirmation. Mastectomy is the treatment of choice in large and recurrent tumors. Wide excision is advised for smaller lesions. Of late breast conservative surgery is being practiced with pre and post operative radiotherapy but results are inconclusive. Over all mastectomy is superior to beast conservative surgery since, it clears the entire tumor with tumor margin. Sarcoma arising from phyllodes tumor will not respond to radiotherapy or chemotherapy. 


\section{References}

[1]. Guillot.E; Countturaud.B; Reyal.F; Curnier.A; Rauinet. J; Lae.M; Blloet.M; Pierga.JY; Salomon.R; Fitoussi.A; breast cancer study of the institute Curie " management of phyllodes breast tumors"; The Breast Journal, 2011; 17 (2):129- 137. . doi:10.1111/j.15244741.2010.01045.x. PMID 21251125. edit.

[2]. World Health Organization. Histologic Typing of Breast Tumors. 2nd edition. Vol. 2 Geneva, Switzerland: WHO; 1981.

[3]. Deen SA, McKee GT, Kissin MW, "differential cytological features of fibroepithelial lesions of the breast"; Diagn cytopathol, 1999; 20 (2) 53-6. doi:10.1002/(SICI)1097-0339(199902)20:2<53::AID-DC1>3.0.CO;2-T. PMID 9951596.

[4]. Tan. PH, Thike. AA, Tan. WJ, Thu. MM, Busmanis. I, Li. H, Chay. YW, Tan MH; "Predicting clinical behaviour of breast phyllodes tumor" a mammogram based on histological criteria and surgical margins. J Clin Path. 2012; 65 (1) 69-76. doi: $10.1136 /$ jclinpath-2011-200368. PMID 22049216.

[5]. Brath RJ Jr.,Wells WA, Mitchell SE, Cole BF; “ A prospective multi institutional study of adjuvant radiotherapy after resection of malignant phyllodes tumor", Ann Surg Oncol, 2009; 16 (8): 2288-94. doi: 10.1245/s10434-009-0489-2. PMID 19424757.

[6]. Lerwill MF. Biphasic lesions of the breast, Semin Diagn Pathol. 2004; $21: 48-56$.

[7]. Nishimura R, Hasebe T, Imoto S, Mukai K. Malignant phylloides tumour with a non-invasive ductal carcinoma component. Virchows Arch. 1998; 432: 89-93.

[8]. Belkacémi Y, Bousquet G, Marsiglia H, et 1 "Phyllodes Tumor of the Breast". Int J Radiat Oncol Biol Phys. 2007; 70 (2): 492.doi:10.1016/j.ijrobp.2007.06.059.PMID 179317 1796.

[9]. Franceschini G, D'Ugo D, Masetti R, Palumbo F, D'Alba PF, Mulè A, Costantini M, Belli P, Picciocchi A. Surgical treatment and MRI in phyllodes tumors of the breast: our experience and review of the literature. Ann Ital Chir. 2005 Mar-Apr; 76(2):127-40. 